\title{
Mapping Study on the Use of Digital Games for Persons with Disabilities in Upper Members
}

\author{
FLÁVIA G. FERNANDES ${ }^{1}$, ALEXANDRE CARDOSO ${ }^{2}$ and RENATO A. LOPES ${ }^{3}$ \\ ${ }^{1}$ Departamento de Computação, Universidade Federal de Goiás/UFG, Av. Dr. Lamartine Pinto \\ de Avelar, 1120, Setor Universitário, Bloco M, 75704-020 Catalão, GO, Brazil \\ ${ }^{2}$ Faculdade de Engenharia Elétrica, Universidade Federal de Uberlândia/UFU, Av. João \\ Naves de Ávila, 2121, Santa Mônica, 38400-902 Uberlândia, MG, Brazil \\ ${ }^{3}$ Faculdade de Computação, Universidade Federal de Uberlândia/UFU, Av. João Naves \\ de Ávila, 2121, Santa Mônica, 38400-902 Uberlândia, MG, Brazil \\ Manuscript received on September 11, 2018; accepted for publication on January 14, 2019
}

\begin{abstract}
How to cite: FERNANDES FG, CARDOSO A AND LOPES RA. 2019. Mapping Study on the Use of Digital Games for Persons with Disabilities in Upper Members. An Acad Bras Cienc 91: e20180936. DOI 10.1590/0001-3765201920180936.

Abstract: Digital games are no longer seen as a form of entertainment detrimental to health. They have become an important tool to improve the treatment of patients, ranging from those who are experiencing a serious illness, such as cancer, to those requiring milder procedures such as physiotherapy. However, there are people who are physically disabled and have difficulty playing digital games. In this line of reasoning, this work presents a systematic review on the use of digital games for people with physical disabilities in the upper limbs. The sources of research were the following indexed databases: IEEE Xplore, Portal of Periodicals of the Coordination of Improvement of Personnel of Higher Level (CAPES), PubMed, Scielo, Science Direct, Scopus and Web of Science. It should be noted that the application of Systematic Mapping in the elaboration of a bibliographical review allowed to identify the main gaps for the development of new research, and to redirect to the main publications related to the study. Finally, the results shows that it is an area that is constantly expanding.
\end{abstract}

Key words: digital games, mapping study, physical disability, upper limbs.

\section{INTRODUCTION}

Often people with physical or mental disabilities are victims of prejudice and discrimination. In addition, there is the custom of not receiving the same type of treatment and have the freedom to come and go harmed by the poor conditions of public and private access routes. However, the Universal Declaration of Human Rights makes it

Correspondence to: Flávia Gonçalves Fernandes

E-mail: flavia.fernandes92@gmail.com

ORCid: https://orcid.org/0000-0001-5077-2226 clear that all people should be treated fraternally, regardless of disability. In the specific case of Brazil, the Federal Constitution defines as a goal the search for the welfare of all, without any kind of discrimination. In the same way, the Brazilian Penal Code determines as punishable the criminal acts and of disrespect caused by discriminatory factors (Rights 2016).

Physical disability can be defined as different motor conditions that affect people by compromising mobility, general motor coordination and speech, as a consequence of neurological, neuromuscular, 
orthopedic, congenital or acquired malformations (Kouroupetroglou 2013).

According to the Salamanca Declaration, human differences are normal and learning must adapt to the needs of children rather than adapt the child to preconceived assumptions about the pace and nature of the learning process.

Moreover, the long time necessary for the promotion of body awareness, acceptance of the disability and the low motivation generated by traditional methods are indicated as a reason for treatment abandonment, characterizing as one of the main causes of therapeutic failure (Dias et al. 2009). Another problem encountered by people with physical disabilities is the lack of resources that allow the continuity of home treatment, with little or no face-to-face monitoring of a therapist (Botella et al. 2010). It is still important to remember that people who are born with congenital malformation of a member of the human body also have other health problems, which may be physical (in other limbs), mental, cardiovascular, respiratory, among others.

Thus, the use of the digital game can become an alternative in providing greater motivation in the games through challenges with virtual techniques, working concepts that can aid in the cognition, in the emotional and physical aspects of the patients, in favoring the movements of the affected limb, during leisure, socialization and coexistence with other people.

In this perspective, the promotion of the corporal consciousness of virtual form through games aims to simulate real situations; it is also perceived that the use of it improves the functionality of affected limbs and leads it to resume activities in the areas of occupational performance (Grande et al. 2011). Therefore, it is important to carry out research whose goal is to make people with physical disabilities also have the benefits of using digital games.
In this context, the objective of this work is to verify the panorama of the related research on the application of digital games for people with physical disabilities in the upper limbs, presenting a systemic study about what has been published for this aspect. Since the number of existing digital games for daily help or treatment of physical or psychological illnesses is very extensive, this work has been restricted to digital games that treat people with physical disabilities in the upper limbs.

\section{METHOLOGY}

For the review of the literature, a mapping study was developed, according to the methodology proposed by (Bailey et al. 2007) and (Petersen et al. 2008), which consists in the search of studies registered in databases through logical operators for the selection of articles, from the selection of key words or expressions.

This systematic mapping technique was also used by (Paula and Ilha 2016, Bonfim et al. 2017, Rodrigues et al. 2017).

The databases considered were: Portal of Periodicals of the Coordination of Improvement of Higher Level Personnel - CAPES (Capes 2018), Scopus (Scopus 2018), Science Direct (Science 2018), Scielo (Scielo 2018), IEEE Xplore (IEEE 2018), PubMed (PubMed 2018) and Web of Science (Web 2018), databases available at the Universidade Federal de Uberlândia. It is noteworthy that only articles from peer-reviewed journals were analyzed.

The logical expressions used were: (a) "digital games" AND "disability" AND "upper limbs" and (b) "digital games" AND "disability" AND ("wearable" OR "virtual reality"). These strings were chosen to search for works that involve digital games for people with physical disabilities in the upper limbs and who use wearable devices for adaptation, rehabilitation or insertion of this public in the social context. 
After the initial search, they were adopted as filters: the language (English and Portuguese); without restriction of the area of knowledge and type of publication (peer-reviewed journal article). When the filters were applied in each database, the articles were classified by the year of publication and the titles, observing the possible repetitions between the databases.

The final selection stage of the articles was made after reading and analyzing of the titles and abstracts, in order to exclude those works that were not directly related to the subject being studied, being the systematic review made from the results obtained.

For this, the criteria used to read the titles and summaries of the articles were: if they contained the keywords, and if they were adherent to the intended context, which in this case refers to research involving digital games for people with physical disabilities upper limbs and/or wearing wearable devices. In this sense, works dealing with different themes were discarded.

\section{RESULTS AND DISCUSSION}

After the searches in the databases, the results were organized in the form of charts and tables with the purpose of presenting them in a more practical way. Table I shows the total results obtained in the mapping and Figure 1 shows the evolution of the publication of the articles of periodicals surveyed, considering the aforementioned keywords and the July 2018 deadline.

Some databases presented a large number of articles repeated among themselves, and twenty articles of these databases that were classified in adherents as to the title and abstract had restricted access to the full text, making it impossible to use them.

Thus, in the final selection, through a systematic mapping, a database was created with 15 (fifteen) database references for the string "Digital games" AND "disability" AND "upper limbs" and 14 (fourteen) references for the string "Digital games" AND "disability" AND ("wearable" OR "virtual reality"), totaling 29 references adhering to the work. The "Digital games" AND "disability" AND "upper limbs" string presented the highest percentage of articles with title and abstract adherents and access to the full text selected after the initial result.

The evolution of the annual publication of the articles selected in the international search can be seen in Figure 1, where it is possible to notice that the articles involving the subject addressed in this work were published in the last decades. In this way, it is observed that the subject is recent and presents the tendency of expansion in the research. One possible explanation for this fact is a greater maturity in the application of digital games in the most diverse areas and in the awareness of the need to include people with physical disabilities.

Periodicals in which articles on these topics have been published are presented in Table II.

Thus, one can notice that the main journals that published on the subject recently were "Disability and Rehabilitation: Assistive Technology" and "Brazilian Journal of Biomedical Engineering". Both are international and multidisciplinary journals.

The first has as mission to promote and share the interdisciplinary and integrative science of assistive technology services for people with disabilities, chronic diseases and challenges for the performance of activities and participation in vital roles, achieve a better functioning and quality of life.

The second objective is to provide didactic material and professional updating, as well as serve as a forum for the establishment of policies for the development and incorporation of health technologies by the public or private sectors. This multidisciplinary journal is aimed at readers and authors interested in using or developing tools based on engineering and physical sciences to 
TABLE I

Results obtained in the searches in the databases.

\begin{tabular}{|c|c|c|c|c|}
\hline \multirow{4}{*}{ DATABASE } & \multicolumn{4}{|c|}{ Keywords } \\
\hline & \multicolumn{2}{|c|}{$\begin{array}{c}\text { "Digital games" AND "disability" } \\
\text { AND "upper limbs" }\end{array}$} & \multicolumn{2}{|c|}{$\begin{array}{l}\text { "Digital games" AND “disability" } \\
\text { AND ("wearable" OR virtual reality }\end{array}$} \\
\hline & \multicolumn{4}{|c|}{ Number of articles } \\
\hline & Initial & With adhering title & Initial & $\begin{array}{c}\text { With } \\
\text { adhering title }\end{array}$ \\
\hline IEEE Xplore & 115 & 14 & 80 & 25 \\
\hline Periódicos Capes & 221 & 17 & 265 & 30 \\
\hline PubMed & 57 & 8 & 50 & 14 \\
\hline Scielo & 20 & 9 & 14 & 10 \\
\hline Science Direct & 187 & 26 & 64 & 20 \\
\hline Scopus & 107 & 14 & 182 & 15 \\
\hline Web of Science & 116 & 7 & 70 & 18 \\
\hline Total & 823 & 95 & 725 & 132 \\
\hline $\begin{array}{l}\text { Total number of articles without replicates } \\
\text { between databases }\end{array}$ & & 36 & & 25 \\
\hline $\begin{array}{l}\text { Number of articles selected after title } \\
\text { reading and abstract }\end{array}$ & & 12 & & 8 \\
\hline
\end{tabular}

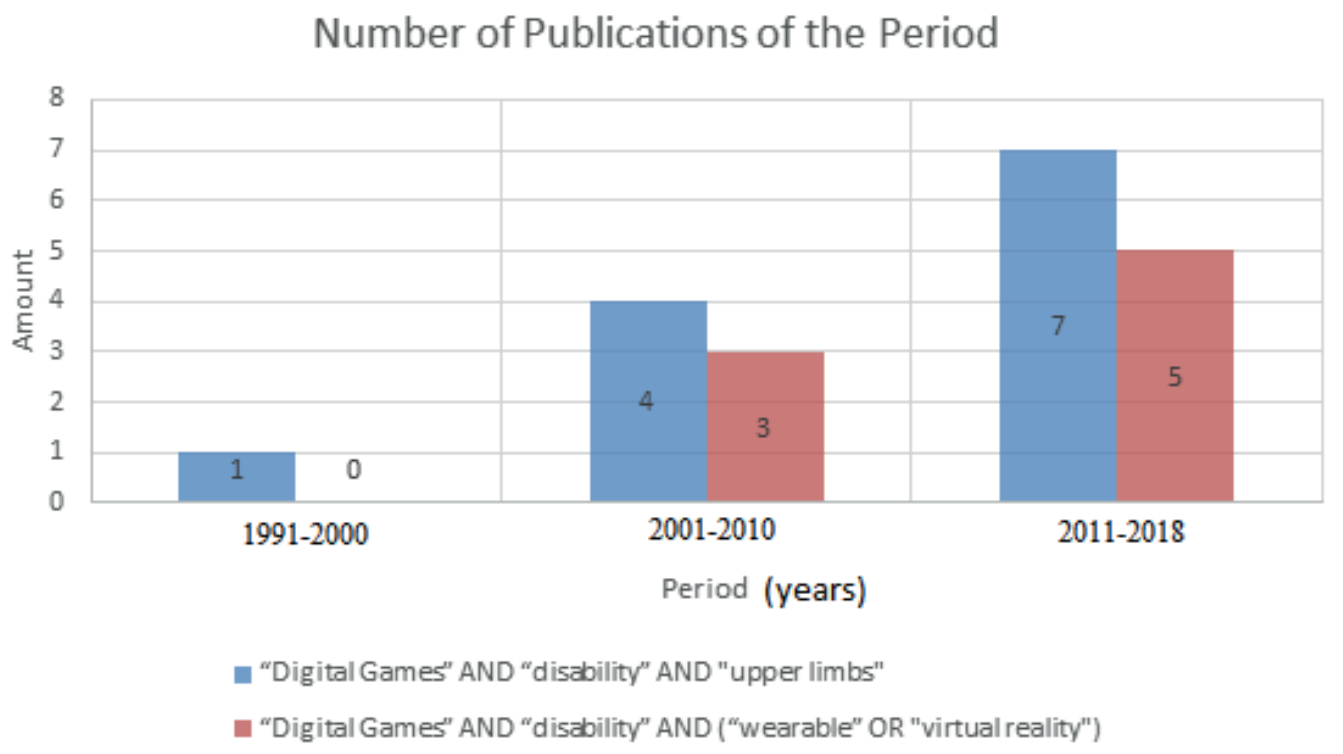

Figure 1 - Evolution of the publication of articles raised according to the keywords used in the mapping. 
TABLE II

Main journals on "digital games for people with physical disabilities in the upper limbs and/or wearing wearable devices".

\begin{tabular}{|c|c|}
\hline Newspaper & $\begin{array}{l}\text { Number } \\
\text { of articles } \\
\text { published }\end{array}$ \\
\hline $\begin{array}{l}\text { Disability and Rehabilitation: Assistive } \\
\text { Technology }\end{array}$ & 3 \\
\hline $\begin{array}{l}\text { Brazilian Journal of Biomedical } \\
\text { Engineering }\end{array}$ & 3 \\
\hline $\begin{array}{l}\text { Internacional Journal of Developmental } \\
\text { Disabilities }\end{array}$ & 2 \\
\hline Biosystems and Biorobotics & 2 \\
\hline $\begin{array}{l}\text { Journal of Pediatric Rehabilitation } \\
\text { Medicine }\end{array}$ & 2 \\
\hline $\begin{array}{l}\text { Journal on Applied System Innovation for } \\
\text { Modern Technology }\end{array}$ & 1 \\
\hline Medical Journal of Malasya & 1 \\
\hline Burns & 1 \\
\hline International Journal of Social Robotics & 1 \\
\hline Trials & 1 \\
\hline $\begin{array}{l}\text { International Journal on Technology and } \\
\text { Innovation in Health }\end{array}$ & 1 \\
\hline $\begin{array}{l}\text { International Journal on Modelling and } \\
\text { Simulation }\end{array}$ & 1 \\
\hline $\begin{array}{l}\text { International Journal Gamification for } \\
\text { Human Integration }\end{array}$ & 1 \\
\hline
\end{tabular}

understand and solve problems in the biological and medical sciences. However, it is observed that there is not a great discrepancy in the quantity of publications between the journals. This can be explained in part by the low number of papers selected in this research.

The Figure 2 shows a bar chart horizontally with the number of journal articles published by country. The works published are from different countries, such as the United States, Germany, Brazil, China, Austria, Italy, France, Japan, and South Korea. In South America, works developed only in Brazil that address this theme have been found.

Subsequently, the 29 articles were read in full related to the subject in question, which will be detailed in the next section of the article. Although they present different scopes, the selected papers contribute to the ratification of the relevance of the use of natural interaction systems to promote upper body awareness or alternative interactions.

\section{SELECTED WORKS}

The PRISMA method was used to select the works. Thus, the works listed below include details about each of them, according to the PRISMA checklist (title, abstract, introduction, methods, results and conclusions).

\section{ARTICLE A1}

The article A1, called the "Game Console Controller Interface for People with Disability," features an interface device that allows people with disabilities in the upper limbs to play console games. This prototype is a completely new device connected between the original controller and the console. It allows the connection of the interface most suitable for the specific deficiency. Thanks to this device, the specific interface acts in the same way as the push buttons and/or joysticks of the original controller. The mapping of functions from external sensors to the original controller is performed by a simple and intuitive procedure (Iacopetti et al. 2008).

\section{ARTICLE A2}

The article A2, "Virtual Reality as Adjunctive Therapy for Upper Limb Rehabilitation in Cerebral Palsies", presents a game that uses Virtual Reality as adjunctive therapy for the rehabilitation of the upper limbs of children victims of cerebral palsy. The level of cooperation and satisfaction of children was assessed when practicing arm and hand movements during play activities in a physical environment compared to a virtual environment based on video capture using five degrees of freedom. Although cooperation was similar in both environments, children expressed greater satisfaction with the physical environment than with the virtual environment. The reasons 


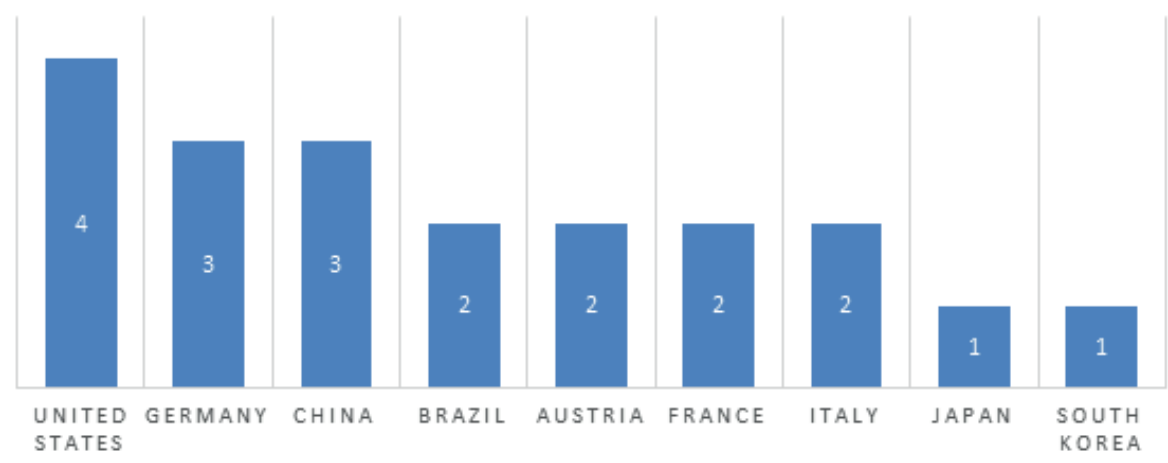

Figure 2 - Number of journal articles selected published by country.

for this may include difficulty or frustration with virtual activities and/or suitability of computer games (Guberek et al. 2009).

\section{ARTICLE A3}

The article A3, "Upper Extremity Rehabilitation of Children with Cerebral Palsy Using Accelerometer Feedback on a Multitouch Display," presents a system for rehabilitation for upper limbs of children with cerebral palsy using accelerometer feedback in a multitouch viewer. The system allows children to engage in interactive gaming scenarios while intensively performing the desired exercises. To encourage correct posture during therapeutic exercises, a wireless kinematic sensor in the patient's trunk was used as a feedback channel for the games. The system went through several design phases, incorporating input from therapy observations and clinical sessions, as well as feedback from medical professionals (Dunne et al. 2010).

\section{ARTICLE A4}

The article A4 "Development of an EMG-ACCBased Upper Limb Rehabilitation Training System" has as its main theme the development of a superior limb rehabilitation training system designed for use by children with cerebral palsy. The authors sought to meet the training requirements at home, leveraging the combination of portable accelerometer sensors and surface electromyography used on the upper limb to capture functional movements. The results of a long-term training conducted with three subjects of cerebral palsy demonstrated that they could improve the performance of the game through repetitive training, requiring persistent training to improve the rehabilitation effect (Liu et al. 2016).

\section{ARTICLE A5}

The article A5, "Pediatric rehabilitation with the reachman's modular handle," presents the results of a preliminary study with a child with cerebral palsy using the ReHaptic Handle, a new robotic device for pediatric rehabilitation of upper limb function. Interactive computer games were implemented to increase participant engagement and engagement, thus promoting motor recovery. Pinching with forefinger and thumb, forearm supination/pronation as well as flexion/extension of the wrist were trained two to three times a week for fifteen minutes each. An increase in the accuracy and smoothness of forearm supination/pronation movement with the subject was observed, as well as a reduction in the duration of movement (Tong et al. 2015).

\section{ARTICLE A6}

The article T6, "Hand Therapist: the rehabilitation approach based on wearable technology and video gaming", is a system of hand rehabilitation, mainly for patients victims of Cerebral Vascular Accident 
(CVA), composed by: Myo clamp, glove robotics and the Unity 3D game engine. This approach presents a solution that combines performance, low cost and motivation for hand therapy (Lipovský et al. 2015).

\section{ARTICLE A7}

The article T7, "Hand Posture and Gesture Recognition using Myo Armband and Spectral Collaborative Representation based Classification", proposes the use of Spectral Domain-based collaborative representation to recognize the postures and gestures of the electromyography (EMG) recordings acquired by a newly introduced sensor: Myo Thalmic Labs clamp. The recognition accuracy obtained for a set of six gestures and postures is promising, with an accuracy greater than $97 \%$, which is an efficient result in related literature. The algorithms were developed for the creation of an intuitive man-machine interface for navigating in a robotic wheelchair (Boyali 2015).

\section{ARTICLE A8}

The article A8, "A strategy to support the humancomputer interaction of children with disabilities in the upper limbs by wearable device", have the goal increasing the use of digital games for the target audience. In this way, digital games were adapted for children with physical disabilities in the upper limbs, either due to congenital or acquired malformations, in order to allow the use of digital games by this public and to help in the acceptance of deficiency. For its development, the functionalities of the Myo wearable device were used to control a puzzle game as a means of providing the interaction between the individual and the game, since Myo is a bracelet that offers ease and practicality of use. Thus, it was offered to these children an additional tool (digital games) for the process of promoting body awareness and increasing their accessibility to games (Fernandes 2017).
In this context, the game is controlled by the movements of the user's upper limb, which presents the deficiency, through Myo.

Thus, in the long term, it can help in the acceptance of motor limitation, motivate patients to use the disabled member more frequently, develop and create abilities, raise potential, learn new technologies, improve cognitive, emotional and physical aspects, socialization and leisure.

\section{ARTICLE A9}

In the article A9, "An Interactive Upper Limb Rehab Device for Elderly Stroke Patients", elderly stroke patients need intensive and taskspecific rehabilitation exercises to improve manual dexterity. Most of the robot-mediated devices used for these exercises currently do not fully motivate them to complete the exercises or measure the progress of the therapy. In this study, we developed an ergonomic, portable upper limb rehabilitation device (QikRehab) with the help of interactive media. Interactive rehabilitation games with options to monitor the therapy progress is the design novelty of this device. Three prototypes on the console design and fabrication are investigated in sequence, which are evolved based on the feedbacks and comments from therapists. Two rapid prototyping (RP) technologies are utilized to fabricate the second and the third prototypes for initial user study and feedback collection. Four exercises including grip, pitch, twist and roll are designed on this console to simulate upper limb motions within activities of daily living. To motivate old adults to exercise more conveniently in either hospital or home, three interactive rehabilitation games (Pig Hole, Rat Hunting and Intensive Grip) are designed to motivate such exercises, the achievement obtained in the games are automatically recorded for movement ability assessment. Furthermore, we presente several discussions on the pilot user testing through a workshop and follow up interview and 
suggest several future improvements of this device (Jie et al. 2017).

\section{ARTICLE A 10}

In the articleA10, "A robotic platform for customized and interactive rehabilitation of persons with disabilities", have developed a multisensor system for rehabilitation and interaction with persons with motor and cognitive disabilities. The system enables them to perform different therapies using multiple modes of interaction (head and body pose, hand gestures, voice, touch and gaze) depending on the type and degree of disability. Through a training process, the system can be customized enabling the definition of patients' own gestures for each sensor. The system is integrated with a range of applications for rehabilitation. Examples of these applications are puzzle solving, mazes and text writing using predictive text tools. The system also provides a flexible and modular framework for the development of new applications oriented towards novel rehabilitation therapies. The proposed system has been integrated in a mobile robotic platform and uses low-cost sensors allowing to perform nonintrusive rehabilitation therapies at home (GomezDonoso 2017).

\section{ARTICLE A11}

In the article A11, "Assistive technologies to overcome sarcopenia in ageing", sarcopenia is an age-related decline in skeletal muscle mass and function that results in disability and loss of independence. It affects up to $30 \%$ of older adults. Exercise (particularly progressive resistance training) and nutrition are key strategies in preventing and reversing declines in muscle mass, strength and power during ageing, but many sarcopenic older adults fail to meet recommended levels of both physical activity and dietary nutrient intake. Assistive technology (AT) describes devices or systems used to maintain or improve physical functioning. These may help sarcopenic older adults to maintain independence, and also to achieve adequate physical activity and nutrition. There is a paucity of research exploring the use of AT in sarcopenic patients, but there is evidence that AT, including walking aids, may reduce functional decline in other populations with disability. Newer technologies, such as interactive and virtual reality games, as well as wearable devices and smartphone applications, smart homes, 3D printed foods, exoskeletons and robotics, and neuromuscular electrical stimulation also hold promise for improving engagement in physical activity and nutrition behaviours to prevent further functional declines. While AT may be beneficial for sarcopenic patients, clinicians should be aware of its potential limitations. In particular, there are high rates of patient abandonment of AT, which may be minimised by appropriate training and monitoring of use. Clinicians should preferentially prescribe AT devices which promote physical activity. Further research is required in sarcopenic populations to identify strategies for effective use of current and emerging AT devices (Scotta et al. 2018).

\section{ARTICLE A12}

In the article A12, "The Nintendo Wii as a tool for neurocognitive rehabilitation, training and health promotion", health professionals have used virtual reality as an aid for several types of treatment. Given that virtual reality systems are expensive and not always available, a more accessible type of virtual reality technology is video games. The Nintendo Wii ${ }^{\mathrm{TM}}$ (NW) is a video game system that uses virtual reality technology which may be used for health promotion. The Nintendo Wii ${ }^{\mathrm{TM}}$ also provides an opportunity for social interaction; thus, it is a promising tool with great potential for the treatment of specific disorders. The aim of this article is to evaluate the ways in which the Nintendo Wii has been used to treat specific disorders or 
to promote cognitive or physical improvements through a review of the literature. The results have shown that the NW is a potentially useful tool in some therapeutic treatments that can be used with people of diverse social statuses and tastes. Despite the positive initial results, further studies are required to provide a better evaluation of video game usage in therapeutic programs (Pessoa et al. 2014).

\section{ARTICLE A13}

In the article A13, "Active Video Game Play in Children With Cerebral Palsy: Potential for Physical Activity Promotion and Rehabilitation Therapies", to evaluate the potential of active video game (AVG) play for physical activity promotion and rehabilitation therapies in children with cerebral palsy (CP) through a quantitative exploration of energy expenditure, muscle activation, and quality of movement. The design is single-group, experimental study. The setting is human movement laboratory in an urban rehabilitation hospital. The participants are children $(\mathrm{N}=17$; mean age $\pm \mathrm{SD}$, $9.43 \pm 1.51 \mathrm{y}$ ) with $\mathrm{CP}$. The intervention: participants played 4 AVGs (bowling, tennis, boxing, and a dance game) (Howcroft et al. 2012).

The main outcome measures is energy expenditure via a portable cardiopulmonary testing unit; upper limb muscle activations via single differential surface electrodes; upper limb kinematics via an optical motion capture system; and self-reported enjoyment via the Physical Activity Enjoyment Scale (PACES).

Like this, moderate levels of physical activity were achieved during the dance (metabolic equivalent for task $[\mathrm{MET}]=3.20 \pm 1.04$ ) and boxing $(\mathrm{MET}=3.36 \pm 1.50)$ games. Muscle activations did not exceed maximum voluntary exertions and were greatest for the boxing AVG and for the wrist extensor bundle. Angular velocities and accelerations were significantly larger in the dominant arm than in the hemiplegic arm during bilateral play. A high level of enjoyment was reported on the PACES ( $4.5 \pm 0.3$ out of 5).

Therefore, AVG play via a low-cost, commercially available system can offer an enjoyable opportunity for light to moderate physical activity in children with CP. While all games may encourage motor learning to some extent, AVGs can be strategically selected to address specific therapeutic goals (e.g., targeted joints, bilateral limb use). Future research is needed to address the challenge of individual variability in movement patterns/play styles. Likewise, further study exploring home use of AVGs for physical activity promotion and rehabilitation therapies, and its functional outcomes, is warranted.

\section{ARTICLE A14}

The article A14, "Motion Rehab AVE 3D: A VRbased exergame for post-stroke rehabilitation", presents Motion Rehab AVE 3D, a serious game for post-stroke reha- bilitation of patients with mild stroke. The aim is offer a new technology in order to assist the traditional therapy and motivate the patient to execute his/her rehabilitation program, under health professional supervision. The game was developed with Unity game engine, supporting Kinect motion sensing input device and display devices like Smart TV 3D and Oculus Rift. It contemplates six activities considering exercises in a tridimensional space: flexion, abduction, shoulder adduction, horizontal shoulder adduction and abduction, elbow extension, wrist extension, knee flexion, and hip flexion and abduction. Motion Rehab AVE 3D also report about hits and errors to the physiotherapist evaluate the patient's progress. A pilot study with 10 healthy participants (61-75 years old) tested one of the game levels. They experienced the 3D user interface in third-person. Our initial goal was to map a basic and comfortable setup of equipment in order to adopt later. All 
the participants $(100 \%)$ classified the interaction process as interesting and amazing for the age, presenting a good acceptance. Soon, our evaluation showed that the game could be used as a useful tool to motivate the patients during rehabilitation sessions. Next step is to evaluate its effectiveness for stroke patients, in order to verify if the interface and game exercises contribute into the motor rehabilitation treatment progress (Trombetta et al. 2017).

\section{ARTICLE A15}

In the article A15, "Postural synergy based design of exoskeleton robot replicating human arm reaching movements", the concept of postural synergy is recently being widely applied to the mechanical design of prosthesis. However, there are few research literatures on the application of postural synergy in the mechanical design of an exoskeletal rehabilitation robot. In this paper, the reaching movements of human arm are analysed by the principal component analysis method and two most significant synergies of the joints of human arm, which can account for more than $80 \%$ of the variation, are extracted. We propose a postural synergy based method to design the kinematic transmission mechanism for a multi-joint upperlimb exoskeletal rehabilitation robot with two actuators. Additionally, the configuration of passive joints and balance weights is put forward to improve the performance of the robot. The postural synergy based design method is formulated for replicating human arm reaching movements. Finally, tests are taken on the prototype to validate the proposed method. Furthermore, the proposed method can be potentially extended to the design of underactuated robotic arm or manipulator which is just required to perform the specific types of movements (Liu et al. 2018).

\section{ARTICLE A16}

In the article A16, "Robotic and Sensor Technology for Upper Limb Rehabilitation", robotic and sensor-based neurologic rehabilitation for the upper limb is an established concept for motor learning and is recommended in many national guidelines. The complexity of the human hands and arms and the different activities of daily living are leading to an approach in which robotic and sensorbased devices are used in combination to fulfill the multiple requirements of this intervention. A multidisciplinary team of the Fondazione Don Carlo Gnocchi (FDG), an Italian nonprofit foundation, which spans across the entire Italian territory with 28 rehabilitation centers, developed a strategy for the implementation of robotic rehabilitation within the FDG centers. Using an ad hoc form developed by the team, 4 robotic and sensor-based devices were identified among the robotic therapy devices commercially available to treat the upper limb in a more comprehensive way (from the shoulder to the hand). Encouraging results from a pilot study, which compared this robotic approach with a conventional treatment, led to the deployment of the same set of robotic devices in 8 other FDG centers to start a multicenter randomized controlled trial. Efficiency and economic factors are just as important as clinical outcome. The comparison showed that robotic group therapy costs less than half per session in Germany than standard individual arm therapy with equivalent outcomes. To ensure access to high-quality therapy to the largest possible patient group and lower health care costs, robot-assisted group training is a likely option (Jakob et al. 2018).

\section{ARTICLE A17}

In the article A17, "Realidad virtual con fines terapéuticos en pacientes con ictus: revisión sistemática", virtual reality (VR) is used in rehabilitation in order to improve functional 
abilities. In recent years, there have been a large number of publications on the use of RV in patients with neurological disease in order to determine if this therapeutic feature provides a better recovery of motor function. In this work, a systematic review was conducted consulting the Cochrane Original databases, Joanna Briggs Connect, Medline / Pubmed, Cinahl, Scopus, Isi Web of Science and Sport-Discus (Viñas-Diz et al. 2016).

We have included articles published in the last 5 years, published in English and / or Spanish, in patients with stroke, and who use RV to improve motor function. Finally, 4 systematic reviews and 21 controlled and / or randomized clinical trials were selected. Most studies aim to improve motor function of the upper limb, and / or improve performance of activities of daily living, although there is also an article aimed at improving the motor function of shorter limb-improving as well as improving the static- dynamic.

Therefore, there is strong scientific evidence of the beneficial effects of VR on upper limb motor recovery in stroke patients. it is necessary to study in depth the changes generated in cortical reorganization, which type of VR system is best used to determine if the results are maintained in the long term and to define which frequencies and intensities of treatment are most adequate.

ARTICLE A18

In the article A18, “Tongue-able interfaces: Prototyping and evaluating camera based tongue gesture input system", tongue-computer interaction techniques create a new pathway between human mind and computer, with particular utility for people with upper limb impairment. The high dexterity and resilience of the tongue make it a good candidate for interacting with computers. This paper introduces a new interaction technique, camera-based tongue computer interface (CBTCI), which employs tongue without any direct physical contact required. Through a two-phase study, the CBTCI was evaluated and its interaction problems were identified and discussed. In the first phase, the performance of the CBTCI prototype was evaluated through two user tests. The participants' behaviors were observed throughout the first phase and analyzed to scaffold the study of the design problems in gesture-based tongue computer interaction. The Phase 2 study investigated the usability problems of CBTCI which were reflected through the user behavior and participants' feedback; specifically the exploration of referential techniques to make users aware of their tongue position and adjust their gesture. Pros and cons of the referential strategies are discussed to foster future assistive tonguecomputer interface design (Niua et al. 2018).

\section{ARTICLE A19}

In the article A19, "Virtual Reality for Neurorehabilitation: Insights From 3 European Clinics", virtual reality for the treatment of motor impairment is a burgeoning application of digital technology in neurorehabilitation. Virtual reality systems pose an opportunity for health care providers to augment the dose of task-oriented exercises delivered both in the clinic, and via telerehabilitation models in the home. The technology is almost exclusively applied as an adjunct to traditional approaches and is typically characterized by the use of gamified exergames which feature task-oriented physiotherapy exercises. At present, evidence for the efficacy of this technology is sparse, with some reviews suggesting it is the same or no better than conventional approaches. The purpose of this article is to provide real-world insights on the adoption of a virtual reality by 3 European clinics in 3 different service delivery models. These include an inpatient setting for Parkinson disease, a kiosk model for pediatric neurorehabilitation, and a home-based telerehabilitation model for neurologic patients. Motivations, settings, requirements for the 
pathology, outcomes, and challenges encountered during this process are reported with the objective of priming clinicians on what to expect when implementing virtual reality in neurorehabilitation (O’Neil et al. 2018).

\section{ARTICLE A20}

The article A20, "Visual feedback framework for rehabilitation of stroke patients", describes an alternative for the rehabilitation of upper and lower limbs using visual feedback to treat the sequelae of stroke. The Framework contains three-dimensional scenarios which require patient immersion in an intuitive exercise routine supervised by the attending therapist, who further corroborates visually the progress of the recovery. This work is also supported by a DTW algorithm. The algorithm is based on the collection of data through tracking sensors and the identification of the exercise patterns performed by the patient. The delivering of a satisfactory response in case the exercise is completed correctly will support the evaluation of the therapist in the advance of the therapy. The experimental results show the execution of the exercises proposed with different users in order to validate the usability of the framework (Semblantes et al. 2018).

\section{COMPARATIVE ANALYSIS OF SELECTED WORK}

The works cited have confirmed that the use of digital games helps to stimulate users to engage in real-world activities. In addition, it is known that the use of natural interfaces is recent and stimulating, since they are natural human elements, almost imperceptible when the user is immersed in the application. Therefore, the Natural User Interfaces (NUI) are used in several analyzed works.

Adaptation is important to broaden the cognitive potential of People with Special Educational Needs (PNEEs), which is one of the great challenges of inclusion work in the school and social context. But even with few resources, it is possible to offer good alternatives to meet the peculiarities of people with physical disabilities by adapting everyday materials and equipment. These adaptations allow people with disabilities to be able to express themselves, to ask questions, to solve problems and to become more participatory, thus allowing greater social interaction with other people.

The relevance is emphasized in environments related to the medical field that require a process of reproduction or repetition of actions or movements that produce a greater motivational scenario than the traditional methodology.

The work A1 features a driver adapted for people with physical disabilities in the upper limbs. However, the A8 work uses a wearable device for user-controlled gesture adaptation, which is something more practical and easy to use, as well as being an innovative and attractive technology for people.

Typical console controllers are equipped with various buttons and joysticks often to be operated at the same time, making it a real barrier. Some do-it-yourself solutions exist adapting business controllers for special needs. However, video game control is completely left to the disabled and often it is still not able to play with its residual features. In addition, such solutions are neither tradable, being uncertified modifications of a commercial device.

In works A2, A3, A4 and A5, applications for children who have had cerebral palsy can be seen, in order to assist them in returning the movements of their upper limbs normally. These prototypes are important, since cerebral palsy is a non-progressive neurological disease caused by disorders of the developing brain. Physical and occupational therapy, if started at an early age, can help minimize complications, such as joint contractures, and may improve limb movement and limb coordination. Although current forms of therapy for children with cerebral palsy are effective in minimizing 
symptoms, many children find them boring or repetitive.

The $\mathrm{A} 6$ and $\mathrm{A} 7$ papers present researches using the wearable device Myo. In A6, the application is a form of hand therapy aimed at patients suffering from stroke, who do not have physical disabilities, but difficulty in handling objects. In this system, the user performs several repetitive exercises to recover the movements of the hand and, in addition to Myo, use a glove with sensors. In A7, the prototype was created for people who use wheelchairs. Thus, the user moves the wheelchair through his own gestures of the arm in which the Myo is placed. In this case, the deficiency of people occurs in the lower limbs. In these two projects, the interaction medium could be switched to Kinect and joystick, respectively, which would probably achieve the same result. However, in A8, these other technological tools could not be used, since people have physical disabilities in the upper limbs and have difficulty using the aforementioned tools.

The works A9, A10, A11, A14, A15 and A16 use robotics as a research methodology. Works A12 and $\mathrm{A} 13$ use video games as a research methodology. Works A12, A17, A18, A19 and A20 love virtual reality as a tool for project development.

Most of the work is aimed at rehabilitation, namely: stroke, cerebral palsy, wheelchair, motor and cognitive, physical / muscular, neurological.

The Table III presents the topics covered in each study, comparing them to each other. The works are specified with acronyms (A1 to A20), obeying the order of presentation of the same ones in the text.

The information presented in Table III leads to the conclusion that, among the selected papers, all of them deal with adaptation of games to upper limbs. The material used for adaptation used for each work was chosen because of adherence to the problem to be solved and informational protection.

The application area of the adaptation, in the great majority of the works, is related to school area, mainly learning and social inclusion, demonstrating the importance of the use of alternative procedures to the traditional ones for the most varied types of treatment. The other works involve creating games for rehabilitation of people with physical disabilities or motor limitation due to cerebral palsy or stroke.

Another aspect of A8, which differentiates it from others, is the motivational characteristic, in which the game benefits from the digital resources to promote greater immersion and interactivity of the people and, consequently, greater motivation of the users to use the application, which causes satisfactory results in the process.

\section{CONCLUSIONS}

Through the mapping, it was possible to verify that there was a growth in the study of the application of digital games for people with physical disabilities in the upper limbs, since it is a form of entertainment and leisure.

Thus, it is important to highlight that the application of systematic mapping in the elaboration of systematic review allows us to identify the main gaps for the development of new researches. In addition, it addresses the main publications linked to the study.

Due to what has been presented in this work, there is a growing interest in researching and publishing in this area related to digital games for people with physical disabilities in the upper limbs or wearing wearable devices.

Initially, the main line of research in this area is aimed at rehabilitation of people with physical disability or who are with cerebral palsy because they have suffered a stroke. However, the current trends are to insert technology more and more into people's daily lives. Therefore, the most worked research methods are geared towards digital interactive games for people with disabilities to help in the process of social, school and digital inclusion. 
TABLE III

Comparation between selected papers.

\begin{tabular}{|c|c|c|c|c|}
\hline Category & Works & Upper Limbs & Lower Limbs & Methods \\
\hline Adapting digital games & A1 & Yes & No & Joystick \\
\hline \multirow{4}{*}{ Game for rehabilitation of cerebral palsy } & $\mathrm{A} 2$ & Yes & No & Virtual Reality \\
\hline & $\mathrm{A} 3$ & Yes & No & Android platform \\
\hline & A4 & Yes & No & Multitouch display \\
\hline & A5 & Yes & No & Robothics \\
\hline Game for AVC rehabilitation & A6 & Yes & No & Мyo \\
\hline Game for wheelchair rehabilitation & A7 & No & Yes & Myo \\
\hline Expansion of the use of digital games & A8 & Yes & No & Myo \\
\hline Game for AVC rehabilitation & A9 & Yes & No & Robothics \\
\hline Motor and cognitive rehabilitation & A 10 & Yes & No & Robothics \\
\hline Game for physical/muscular rehabilitation & A11 & Yes & Yes & Robothics \\
\hline Games for neurological rehabilitation & A 12 & Yes & No & Nintendo Wii, Virtual Reality \\
\hline Game for rehabilitation of cerebral palsy & A13 & Yes & No & Active Video Game \\
\hline Game for AVC rehabilitation & A14 & Yes & Yes & Robothics \\
\hline Physical/muscular rehabilitation & A15 & Yes & Yes & Robothics \\
\hline Game for neurological rehabilitation & A16 & Yes & No & Robothics \\
\hline Games for neurological rehabilitation & A17 & Yes & No & Virtual Reality \\
\hline Game for neurological rehabilitation & A18 & Yes & No & Virtual Reality \\
\hline Game for neurological rehabilitation & A19 & Yes & No & Virtual Reality \\
\hline Game for AVC rehabilitation & $\mathrm{A} 20$ & Yes & No & Virtual Reality \\
\hline
\end{tabular}

With this, it is noticed that the use of natural interface (NUI) is a tendency to assist in the process of inclusion of people with disabilities, such as virtual reality and Myo wearable device, for example, since they are new and more attractive techniques, since they explore the user's own movements in real time. Therefore, there is room for research into new devices capable of offering better interaction between people with disabilities and digital games.

It is also important to note that wearable devices are innovative assistive technologies and can aid in patient rehabilitation, physiotherapy, disability acceptance, leisure, entertainment, adaptation and social inclusion.

Thus, a resource still little used identified from the research done are geared towards the use of wearable devices in digital games for people with physical disabilities and that could have good results in this area due to its practicality and ease of use.

In addition, despite an increase in the number of published works addressing the theme of this research, it is observed that it is a small number in view of the number of people and different situations that need to benefit from the use of digital games.

Another shortcoming is the lack of metrics or protocols to measure the gains or benefits obtained by people with disabilities when using digital games. It is noteworthy that, as already mentioned, some of these people have additional deficiencies, such as mental disabilities.

Therefore, the need to foster this area of research to offer this physically disabled public access to the digital game as a form of treatment, acquisition of knowledge, motivation, entertainment or even by inclusion. In this way, it will be possible to obtain a 
greater maturity in the obtained results and thus to promote a systematization in the use of the digital games in the aid of the promotion of the well-being of these people.

\section{AUTHOR CONTRIBUTIONS}

In this paper, the author Flávia Gonçalves Fernandes is responsible for the research and all the practical procedures performed, which were presented for the defense of her master's dissertation in Biomedical Engineering. Therefore, the writing of the article is also her responsibility. Co-authors Alexandre Cardoso and Renato de Aquino Lopes are, respectively, guiding and coordinating the research project, contributing with suggestions, tips, guidelines and corrections to improve the work.

\section{REFERENCES}

BAILEY J, BUDGEN D, TURNER M, KITCHENHAM B, BRERETON P AND LINKMON S. 2007. Evidence relating to Object-Oriented software design: A survey. First International Symposium on Empirical Software Engineering and Measurement. Computer Society.

BONFIM WB, BALDIN V, PEREIRA RR AND PAULA HM. 2017. Residual sludge from concrete plants: characteristics and applications in the construction of blocks. Elect Civil Eng Jour 13(2): 32-43.

BOTELLA C ET AL. 2010. Treating cockroach phobia with augmented reality. Behavior Therapy 41(3): 401-413.

BOYALI A, HASHIMOTO N AND MATSUMOTO O. 2015. Hand Posture and Gesture Recognition using Myo Armband and Spectral Collaborative Representation based Classification. IEEE $4^{\text {th }}$, Glob.

CAPES - COORDENAÇÃO DE APERFEIÇOAMENTO DE PESSOAL DE NÍVEL SUPERIOR. Portal de Periódicos. 2018. Disponível em: http://www-periodicos-capes-govbr.ez49.periodicos.capes.gov.br/. Acesso em 15 de outubro de 2018.

DIAS RS, SAMPAIO ILA AND TADDEO LS. 2009. Physiotherapy: the introduction of playfulness in the process of rehabilitation of patients under physiotherapeutic treatment. In: VIII Brazilian Symposium on Games and Digital Entertainment, 4, Rio de Janeiro, RJ.

DUNNE A ET AL. 2010. Upper Extremity Rehabilitation of Children with Cerebral Palsy using Accelerometer Feedback on a Multitouch Display. $32^{\text {nd }}$ Annual
International Conference of the IEEE EMBS, Buenos Aires-Argentina.

FERNANDES FG. 2017. A strategy to support the humancomputer interaction of children with disabilities in the upper limbs by wearable device. 2017. Tese de Mestrado. Universidade Federal de Uberlândia, MG, 152 p. (Unpublished).

GOMEZ-DONOSO F. 2017. A robotic platform for customized and interactive rehabilitation of persons with disabilities. Pattern Recognit Lett 99: 105-113.

GRANDE AAB, GALVÃO FRO AND GONDIM LCA. 2011. Virtual rehabilitation through videogame: case report in the treatment of a patient with high lesion on the median and ulnar nerves. Mag Acta Fis 18(3): 157-162.

GUBEREK R ET AL. 2009. Virtual Reality as Adjunctive Therapy for Upper Limb Rehabilitation in Cerebral Palsy. Virtual Rehabilitation International Conference.

HOWCROFT J ET AL. 2012. Active Video Game Play in Children With Cerebral Palsy: Pot. for Phys. Arch Phys Med Rehabil 93(8): 1148-1456.

IACOPETTI F ET AL. 2008. Game Console Controller Interface for People with Disability. Comp Soc IEEE, p. 757-762.

IEEE Xplore. 2018. Available at: https://ieeexplore.ieee.org/ Xplore/home.jsp/. Accessed on October 15, 2018.

JAKOB I ET AL. 2018. Robotic and Sensor Technology for Upper Limb Rehabilitation. Innovations Influencing Physical Medicine and Rehabilitation. PM R 10: 189-197.

JIE S ET AL. 2017. An Interactive Upper Limb Rehab Device for Elderly Stroke Patients. Procedia Elsevier CIRP 60: 488-493.

KOUROUPETROGLOU G. 2013. Disability Informatics and Web Accessibility for Motor Limitations. IGI Global.

LIPOVSKÝ R AND FERREIRA HA. 2015. Hand Therapist: a rehabilitation approach based on wearable technology and video gaming. Portuguese BioEngineering Meeting, 4, Porto: Portugal.

LIU K ET AL. 2018. Postural synergy based design of exoskeleton robot replicating human arm reaching movements. Rob Auton Syst 99: 84-96.

LIU L ET AL. 2016. Development of an EMG-ACC-Based Upper Limb Rehabilitation Training System. IEEE Transactions on Neural Systems and Rehabilitation Engineering.

NIUA S ET AL. 2018. Tongue-able interfaces: Prototyping and evaluating camera based tongue gesture input system. Smart Health, p. 25-34.

O'NEIL O ETAL. 2018. Virtual Reality for Neurorehabilitation: Insights From 3 European Clinics. Innovations Influencing Physical Medicine and Rehabilitation. PM R 10: 198-206.

PAULA HMD AND ILHA MSDO. 2016. Use of moringa oleifera in the treatment of wastewater from concrete 
plants: systematic mapping. Elect Civil Eng Jour 11(1): 50-60.

PESSOA TM ET AL. 2014. The Nintendo Wii as a tool for neurocognitive rehabilitation, training and health promotion. Comp in Hum Behav 31: 384-392.

PETERSEN K, FELDT R, MUJTABA S AND MATTSSON M. 2008. Systematic Mapping Studies in Software Engineering. School of Eng., Blekinge Institute of Technology. University of Bari, Italy.

PUBMED. 2018. Available at: https:/www.ncbi.nlm.nih.gov/ pubmed/. Accessed on October 15, 2018.

RIGHTS GUIDE. Discrimination with disabled people. 2016. Available at: http://www.guiadedireitos.org/index. php?option $=$ com_content $\&$ view $=$ article $\&$ id $=1040 \&$ Item $\mathrm{id}=264$. Accessed on August 20, 2018.

RODRIGUES KC, MESQUITA HC, EDUARDO RC AND PAULA HM. 2017. Systematic mapping of references in the use of BIM in the compatibilization of projects in the civil construction. Electr Civil Eng Jour 13(1): 219-239.

SCIELO. 2018. Available at: www.scielo.org/. Accessed on October 15, 2018.
SCIENCE DIRECT. 2018. Available at: http://www. sciencedirect.com/. Accessed on October 15, 2018.

SCOPUS. 2018. Available at: https://www.scopus.com/. Accessed on October 15, 2018.

SCOTTA RAET AL. 2018. Assistive technologies to overcome sarcopenia in ageing. Maturitas 112: 78-84.

SEMBLANTES PA ET AL. 2018. Visual feedback framework for rehabilitation of stroke patients. Inf in Med Unloc 13: 41-50.

TONG LZ ET AL. 2015. Pediatric rehabilitation with the reachMAN's modular handle. Procedings IEEE Eng Med Biol Soc 2015: 3933-3936.

TROMBETTA M ET AL. 2017. Motion Rehab AVE 3D: A VR-based exergame for post-stroke rehabilitation. Comp Meth Prog 151: 15-20.

VIÑAS-DIZ S ET AL. 2016. Realidad virtual con fines terapéuticos en pacientes con ictus: revisión sistemática. Neurologia 31(4): 255-277.

WEB OF SCIENCE. 2018. Available at: https:/clarivate.com/ products/web-of-science/. Accessed on October 15, 2018. 